\title{
The Effects of Horse-riding Simulator Training with Dual-task on Pulmonary Function and Flexibility in Healthy Adults
}

\author{
Jeong Pyo Seo', Yoon Tae Hwang², Jung-Won Kwon \\ 'Department of Physical Therapy, College of Health Sciences, Dankook University, Cheonan, Republic of Korea; ${ }^{2}$ Department of Physical Therapy, \\ Gangneung Yeongdong University, Gangneung, Republic of Korea
}

Purpose: The purpose of the present study was to investigate the effects of a simultaneous dual-task and horse-riding simulator (HRS) training regime on pulmonary function and flexibility.

Methods: Sixteen subjects were recruited and randomly allocated to two groups: a dual-task (DT) $(n=8)$ or a single-task (ST) $(n=8)$ training group. Flexibility and pulmonary function were assessed before and after HRS training. Both groups underwent HRS training for 4 weeks, 3 times/week in 15-minute training sessions. The ST group underwent HRS training and the DT group underwent dual-task HRS training, which consisted of throwing and catching a ball and ring catching while HRS training.

Results: Training significantly increased flexibility and FVC (forced vital capacity) and FEV1 (forced expiratory volume in 1 second) in both groups ( $p<0.05)$, but FEV1/FVC and PEF (peak expiratory flow) were not significantly different after training in both groups $(p>0.05)$. After the training, flexibility and FVC in the DT group were significantly greater than in the ST group $(p<0.05)$, but FEV1, FEV1/ $\mathrm{FVC}$, and PEF were not significantly different ( $p>0.05$ ).

Conclusion: Simultaneous dual-task and HRS motor training improved flexibility, FVC, and FEV1, and our comparative analysis suggests that dual-task HRS training improved flexibility and FVC more than single-task training.

Keywords: Horse-riding simulator, Dual task, Pulmonary function

\section{INTRODUCTION}

Hippotherapy is a therapy with positive physical, social, and mental impacts. The rhythm of a horse walking is similar to that of normal gait and provides feelings of comfort and security. ${ }^{1}$ Hippotherapy attracts attention as a new treatment method to improve gait and balance. It gives comfort and satisfaction to feel the rhythms on horseback and can be beneficial for mental health. ${ }^{2,3}$ Therefore, hippotherapy is used in rehabilitation as well as sports or entertainment. ${ }^{4-6}$ Hippotherapy is known to be effective in enhancing the three-dimensional movements of subjects using the horse as a tool, as well as inducing responses to the movements of the horse, reducing abnormal muscle tension, improving patterns of movement, strengthening muscles, adjusting trunk balance, and improving gait. ${ }^{7,8}$ Hippotherapy also has positive effects on patients with a central nervous system or musculoskeletal injury and has been used to treat cerebral pal-

Received Nov 27, 2020 Revised Dec 4, 2020

Accepted Dec 8, 2020

Corresponding author Jung-Won Kwon

E-mail wnddnjs103@naver.com sy, ${ }^{9}$ multiple sclerosis, ${ }^{10}$ spinal cord injuries, ${ }^{11}$ strokes ${ }^{12}$ and intellectual disabilities. ${ }^{13}$ Furthermore, it has also been used to improve balance, core stability, lower limb muscle strength, and gait speed. ${ }^{9-11}$ However, the technique is limited by low accessibility, fall risk, and cost, and as a result, a horse-riding simulator (HRS) was developed to simulate horse movements. Training programs based on these simulators have also been reported to improve balance, muscle strength, motion skills, and gait speed, ${ }^{12}$ which indicates both hippotherapy and HRS training have similar significant interventional benefits on physical function. In addition, recent studies have reported that HRS enhances cardiopulmonary functions and has the same effects as aerobic training on healthy adults and children. ${ }^{13,14}$

Dual-task training involves the performance of two tasks simultaneously, and the benefits of this type of training are believed to be derived from competition for the same type of information for processing re-

Copylight (C2020 The Korean Society of Physical Therapy

This is an Open Access article distribute under the terms of the Creative Commons Attribution Non-commercial License (https:// creativecommons.org/license/by-nc/4.0.) which permits unrestricted non-commercial use, distribution, and reproduction in any medium, provided the original work is properly cited. 
sources in the brain. ${ }^{15}$ Clinical studies have shown this type of training enhances performance automatization of motor skills, ${ }^{16,17}$ postural stability, ${ }^{18}$ and gait performance, ${ }^{19}$ and researchers often use dual-task training to improve motor skills and multitasking activities or train executive functions. Dual-task training is generally divided into cognitive and motor dual-tasks. Previous studies have focused on cognitive dual-task training to address cognitive-motor tasks or enhance gait automatization. ${ }^{20,21}$ Recent studies have focused on the use of motor dual-task training to improve executive function. Studies show that dual-task motor training improves balance ability in the elderly and gait performance and gait variability in Parkinson's patients. ${ }^{22,23}$ Furthermore, it has been reported that multicomponent-task training may improve balance and muscle strength more than single-task training. ${ }^{24}$

Several studies have investigated the benefits of HRS training in musculoskeletal and neurological patients, but to the best of our knowledge, few studies have investigated the effects of dual-task HRS training on the pulmonary system or flexibility. Thus, the present study was undertaken to investigate the effects of simultaneous dual-task and HRS motor training on pulmonary function and flexibility.

\section{METHODS}

\section{Subjects}

A total of 16 adults ( 5 men and 11 women with ages ranging from 20 to 29 years) were voluntarily recruited at a local university. These 16 subjects were randomly allocated to either a dual-task (DT) or a single-task (ST) training group, both groups underwent HRS training. Demographic and clinical data were collected, and all subjects provided signed informed consent. The inclusion criteria applied were as follows: 1) no history of musculoskeletal, neurologic, or cardiorespiratory disease, 2) no vestibular or balance disorder, and 3) healthy non-smoking adults.

\section{Experimental methods}

1) Sit and reach test

A Sit-and-reach test was conducted to measure flexibility. A subject sat with knees straight and feet $10 \mathrm{~cm}$ apart. The subjects were then instructed to hold knees steady, to stretch out their arms, and bend over as much as possible for 5 seconds. The maximum distance was recorded before and after the bending.

\section{2) Spirometry}

Before measuring pulmonary function, the equipment was calibrated according to the manufacturer's guidelines (COSMED, Pony FX). Spirometry was performed with a subject wearing a nose clip in a sitting position with knees and hips flexed at $90^{\circ}$. The subject was asked to blow into the mouthpiece of a spirometer as forcefully and quickly as possible and to continue blowing for 6 seconds. Forced vital capacity (FVC), forced expiratory volume in 1 second (FEV1), FEV1/FVC, and peak expiratory flow (PEF) were determined. Measurements were repeated 3 times with a 5 -minute break between measurements and highest values were used in the analysis. FEV1/FVC ratios were calculated by using highest recorded FEV1 and FVC values.

\section{3) Horse-riding simulator}

The JOBA ${ }^{\circledR}$ simulator (Panasonic Electric Works, Osaka, Japan) was used for HRS training. The training involved movements of the whole body in all directions including rotations around lumbar, pelvic, and lower extremities. All subjects sat on the machine, which was operated in "very strong" mode $(0.73 \mathrm{~Hz})$ for 15 minutes, and were required to hold the handle and maintain a lumbar/knee angle of 90 degrees during HRS training.

\section{4) Dual-task training}

Dual-task training consisted of throwing and receiving balls and catching a ring during HRS training. The dual-task training was conducted over 15 minutes sessions, which included 5 minutes of HRS training, 5 minutes of HRS plus ball throwing/catching, and 5 minutes of HRS plus ring catching. A $50 \mathrm{~g}$ rubber ball the size of a soccer ball was used, and subjects threw and received the ball from the experimenter from a distance of 2 meters. The ring used was circular, of diameter $15 \mathrm{~cm}$, and was delivered by the experimenter from a distance of $30 \mathrm{~cm}$ in different directions (Figure 1).

\section{Procedure}

Flexibility and pulmonary function were evaluated using the sit and reach test and by spirometry before HRS training. Subjects in the dual-task (DT) and single-task (ST) groups underwent HRS training for 4 weeks, 3 times per week for 15 minutes per session. Subjects in the ST group only underwent HRS training, and subjects in the DT group underwent the simultaneous dual-task and HRS training regime. Sit and reach, and spirometry testing were conducted after training sessions. 


\section{Statistical analysis}

General characteristics of the subjects were analyzed using descriptive statistics. Shapiro-Wilk test was performed to analyze differences between the two groups in terms of age, height, and weight. The paired t-test was used to compare flexibility and pulmonary function before and after HRS training, and the independent t-test was used for the intergroup analysis. Analysis was performed using change value in the analysis between groups before and after measurement. The analysis was conducted using SPSS Ver. 21.0 (IBM SPSS Statistics for Windows, IBM Corp., Armonk, NY, USA). Null hypotheses of no differences were rejected for $\mathrm{p}$-values of $<0.05$.

\section{RESULTS}

No significant difference was observed between ages, heights, or weights in the DT and ST groups ( $\mathrm{p}>0.05$ )(Table 1). Shapiro-Wilk test showed no significant violation of normal distribution among the outcome measures. Table 2 summarizes group flexibility and pulmonary function results obtained before and after training. In both groups, flexibility, FVC, and FEV1 were significantly increased by training $(\mathrm{p}<0.05)$, but FEV1/FVC and PEF were not $(\mathrm{p}>0.05)$. However, intergroup comparisons showed in-

Table 1. The data for the flexibility and pulmonary function before and after training.

\begin{tabular}{lcc}
\hline Characteristics & DT & ST \\
\hline Age $(\mathrm{yr})$ & $23.8 \pm 1.4$ & $23.6 \pm 0.9$ \\
Gender (M/F) & $2 / 6$ & $3 / 5$ \\
Height $(\mathrm{cm})$ & $166.0 \pm 11.5$ & $168.8 \pm 6.9$ \\
Weight $(\mathrm{kg})$ & $61.3 \pm 15.2$ & $64.8 \pm 8.5$ \\
\hline
\end{tabular}

Mean \pm SD.

DT: dual-task training group, ST: single-task training group.
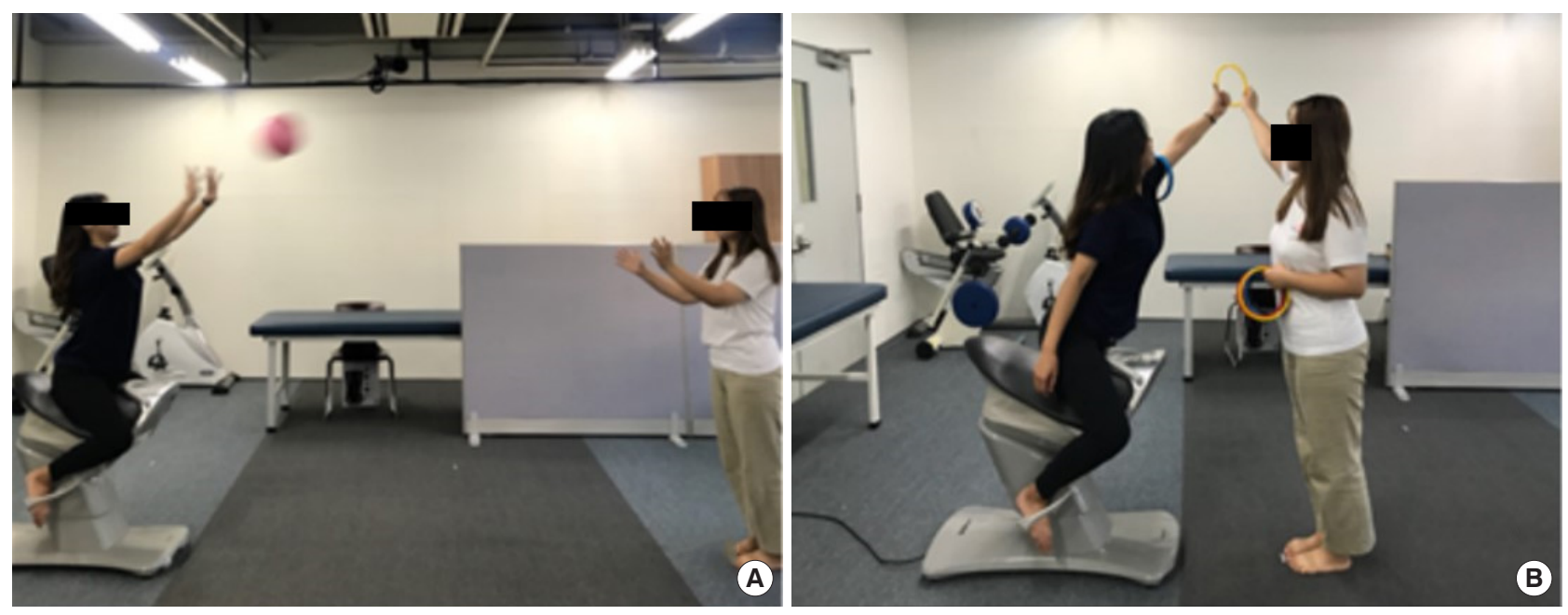

Figure 1. Horse riding simulator training methods with dual-task. (A) Throwing and receiving a ball, (B) Catching a ring.

Table 2. The data for the flexibility and pulmonary function before and after training.

\begin{tabular}{|c|c|c|c|c|c|c|}
\hline & Group & Pre-test & Post-test & $\mathrm{p}$ & Difference & $p$ \\
\hline \multirow[t]{2}{*}{ Flexibility (cm) } & DT & $4.70(11.33)$ & $13.26(9.66)$ & $<0.001^{*}$ & 8.56 (2.94) & $<0.001^{+}$ \\
\hline & ST & $4.39(5.18)$ & $8.96(4.97)$ & $<0.001^{*}$ & $4.58(0.86)$ & \\
\hline \multirow[t]{2}{*}{$\mathrm{FVC}(\mathrm{L})$} & DT & $3.95(1.27)$ & $4.18(1.31)$ & $<0.001^{*}$ & $0.23(0.11)$ & $0.027^{+}$ \\
\hline & ST & $3.77(0.90)$ & $3.87(0.93)$ & $0.027^{*}$ & $0.11(0.11)$ & \\
\hline \multirow[t]{2}{*}{ FEV1 (L) } & DT & $3.29(1.03)$ & $3.47(0.88)$ & $0.011^{*}$ & $0.22(0.29)$ & $0.032^{+}$ \\
\hline & ST & $3.23(0.77)$ & $3.29(0.80)$ & $0.032^{*}$ & $0.06(0.06)$ & \\
\hline \multirow[t]{2}{*}{ FEV1/FVC (\%) } & DT & $84.70(8.93)$ & $86.53(5.29)$ & 0.489 & $1.83(7.11)$ & 0.320 \\
\hline & ST & 86.37 (11.63) & $85.33(9.94)$ & 0.320 & $-1.04(2.75)$ & \\
\hline \multirow[t]{2}{*}{$\operatorname{PEF}(\mathrm{L})$} & DT & $6.74(3.24)$ & $7.53(2.47)$ & 0.106 & $0.79(1.20)$ & 0.145 \\
\hline & ST & $6.66(2.31)$ & $7.21(2.62)$ & 0.145 & $0.55(0.95)$ & \\
\hline
\end{tabular}

Mean \pm SD.

DT: dual-task training group, ST: single-task training group, FVC: forced vital capacity, FEV1: first second of forced expiration volume, PEF: peak expiratory force. * significant difference within group. ${ }^{+}$significant difference between group. $p<0.05$. 
creases in flexibility and FVC were significantly greater in the DT group $(\mathrm{p}<0.05)$, but that increases in FEV1, FEV1/FVC, and PEF in the two groups were not significantly different $(\mathrm{p}>0.05)$.

\section{DISCUSSION}

The present study was performed to determine whether simultaneous dual-task/HRS training improves flexibility and pulmonary function as compared with HRS training. After intervention, flexibility, FVC, and FEV1 increased in both groups, but the DT group obtained significantly greater benefit in terms of flexibility and FVC, which suggests HRS/dual task training can improve flexibility and pulmonary function more than HRS training.

Both groups showed significant flexibility increases after HRS training. Kang et al. ${ }^{25}$ showed that HRS training could increase trunk flexion and improve flexibility. In addition, our results concur with a previous study, in which it was found that HRS training improved flexibility, muscle strength, and muscle endurance in female college students. ${ }^{26}$ It is believed that these benefits are due to the use of the quadriceps femoris and stretching the lower back to maintain balance. According to a previous study, static and dynamic balance abilities were improved by dual-task training in children with cerebral palsy, and the authors suggested that HRS training stretched paraspinal muscles as they are repeatedly contracted and relaxed to maintain balance on an unstable surface..$^{27}$ In addition, we also incorporated ring catching and ball throwing/catching during HRS training to increase exercise intensity. These results and those of the present study suggest that stretch-based exercises can increase flexibility. The aim of applying dual task was to induce sustained concentration during training and encourage user access. Previous studies have shown that a single task can contribute to suboptimal-controlled performance with less attention (Type 3,4), but offered a continuous and diverse set of tasks, such tasks are perceived as critical components and transferred to open-automated performance (Type 1,2). ${ }^{28,29}$

As regards the effect of training on pulmonary function, both groups in the current study, showed significant increases in FVC and FEV1. In a previous study, the effects of HRS training on cardiopulmonary functions were quantitatively measured and assessed the training as low-medium intensity of training. ${ }^{30}$ It was found HRS training increased maximal oxygen consumption (VO2max) and metabolic equivalents of task (MET) and was equivalent to low-intensity exercise, and suggested that it be considered an alternative to aerobic exercise. ${ }^{31}$ Compared to the previous re- search, we agree that HRS training showed progress in FVC and FEV1 because it could be a low-intensity aerobic exercise. In the present study, HRS training had a positive impact on FVC and FEV1, but not on FEV1/FVC or PEF. We found that FEV1/FVC of DT group is $84.70 \pm 8.93 \%$ and ST group $\mathrm{FEV1} / \mathrm{FVC}$ is $86.37 \pm 11.63 \%$, with all normal pulmonary functions, and PEF is a sensitive indicator that changes with the consumption effort of the subject. ${ }^{32}$ We also compared training-induced changes in pulmonary function in the DT and ST groups, and found a significant difference for FVC. In a previous study, HRS training increased VO2max and MET, and the authors suggested VO2max and MET depend on the level of HRS training. ${ }^{30}$ Based on the study of correlation between FVC and VO2max, ${ }^{33}$ we believe FVC change in the DT group was greater because the training regime required additional tasks. ${ }^{34}$

The present study has several limitations that warrant consideration. First, the subjects recruited were in their 20 seconds, which limits the generalizability of our findings. Second, the intervention period of the present study relatively short at 4 weeks. Third, electromyography was not performed on primary or accessory respiratory muscles. We suggest this topic be addressed by further study. Summarizing, flexibility, FVC, and FEV1 were improved in our young adult cohort by HRS training, and our intergroup comparisons showed the dual-task HRS motor training regime improved flexibility and FVC more than HRS training.

\section{REFERENCES}

1. Koca TT, Ataseven H. What is hippotherapy? The indications and effectiveness of hippotherapy. North Clin Istanb. 2015;2(3):247.

2. Potter JT. Therapeutic horseback riding. J Am Vet Med Assoc. 1994; 204:131-3.

3. Debuse D, Chandler C, Gibb C. An exploration of german and british physiotherapists' views on the effects of hippotherapy and their measurement. Physiother Theory Pract. 2005;21(4):219-42.

4. Araujo TB, Silva NA, Costa JN et al. Effect of equine-assisted therapy on the postural balance of the elderly. Rev Bras Fisioter. 2011;15(5):414-9.

5. Beinotti F, Correia N, Christofoletti G et al. Use of hippotherapy in gait training for hemiparetic post-stroke. Arq Neuropsiquiatr. 2010;68(6): 908-13.

6. Debuse D, Chandler C, Gibb C. An exploration of German and British physiotherapists' views on the effects of hippotherapy and their measurement. Physiother Theory Pract. 2005;21(4);219-42.

7. Giagazoglou P, Arabatzi F, Dipla K et al. Effect of a hippotherapy intervention program on static balance and strength in adolescents with intellectual disabilities. Res Dev Disabil. 2012;33(6):2265-70.

8. Zadnikar M, Kastrin A. Effects of hippotherapy and therapeutic horseback riding on postural control or balance in children with cerebral palsy: a meta-analysis. Dev Med Child Neurol. 2011; 53(8):684-91. 
9. Janura M, Peham C, Dvorakova T et al. An assessment of the pressure distribution exerted by a rider on the back of a horse during hippotherapy. Hum Mov Sci. 2009;28(3):387-93.

10. Sterba JA. Does horseback riding therapy or therapist-directed hippotherapy rehabilitate children with cerebral palsy? Dev Med Child Neurol. 2007;49(1):68-73.

11. Silkwood Sherer DJ, Killian CB, Long TM et al. Hippotherapy-an intervention to habilitate balance deficits in children with movement disorders: A clinical trial. Phys Ther. 2012;92(5):707-17.

12. Hilliere C, Collado Mateo D, Villafaina S et al. Benefits of hippotherapy and horse riding simulation exercise on healthy older adults: A systematic review. PM\&R. 2018;10(10):1062-72.

13. Shimomura K, Murase N, Osada T et al. A study of passive weight-bearing lower limb exercise effects on local muscles and whole body oxidative metabolism: A comparison with simulated horse riding, bicycle, and walking exercise. Dyn Med. 2009;8(1):4.

14. Rigby BR, Papadakis Z, Bane AA et al. Cardiorespiratory and biomechanical responses to simulated recreational horseback riding in healthy children. Res Q Exerc Sport. 2015;86(1):63-70.

15. Wickens CD. Multiple resources and performance prediction. Theor Issues Ergon Sci. 2002;3(2):159-77.

16. Beilock SL, Carr TH. On the fragility of skilled performance: What governs choking under pressure? J Exp Psychol. 2001;130(4):701.

17. Ghai S, Driller MW, Masters RS. The influence of below-knee compression garments on knee-joint proprioception. Gait posture. 2018;60:258 61.

18. Resch JE, May B, Tomporowski PD et al. Balance performance with a cognitive task: A continuation of the dual-task testing paradigm. J Athl Train. 2011;46(2):170-5.

19. Schaefer S, Jagenow D, Verrel J et al. The influence of cognitive load and walking speed on gait regularity in children and young adults. Gait posture. 2015;41(1):258-62.

20. Boisgontier MP, Beets IA, Duysens J et al. Age-related differences in attentional cost associated with postural dual tasks: Increased recruitment of generic cognitive resources in older adults. Neurosci Biobehav Rev. 2013;37(8):1824-37.

21. Godde B, Voelcker Rehage C. Cognitive resources necessary for motor control in older adults are reduced by walking and coordination training. Front Hum Neurosci. 2017;11:156.

22. Zanotto T, Bergamin M, Roman F et al. Effect of exercise on dual-task and balance on elderly in multiple disease conditions. Curr Aging Sci. 2014;7(2):115-36

23. Yang YR, Cheng SJ, Lee YJ et al. Cognitive and motor dual task gait training exerted specific training effects on dual task gait performance in individuals with parkinson's disease: A randomized controlled pilot study. PloS one. 2019;14(6):e0218180.

24. Cadore EL, Casas-Herrero A, Zambom-Ferraresi F et al. Multicomponent exercises including muscle power training enhance muscle mass, power output, and functional outcomes in institutionalized frail nonagenarians. Age. 2014;36(2):773-85.

25. Kang SR, Kim UR, Jeong GY et al. Analysis on the flexibility and muscle function in young adults using indoor horseback riding machine. J of RWEAT. 2013;7(1):1-11.

26. Lee SK, Jeong JH. The effects of indoor horseback-riding exercise on health-related fitness, serum lipids, and defecation satisfaction of female collegiate students. Korean J Sport Sci. 2005;16(3):153-60.

27. Kwon H. Changes in the balance and activities of daily living on children with ataxic cerebral palsy from dual task training: Case study. KSIM. 2016;4(4):91-100.

28. Furley P, Schweizer G, Bertrams A. The two modes of an athlete: dualprocess theories in the field of sport. Int Rev Sport Exerc Psychol. 2015; $8(1): 106-24$.

29. Carson HJ, Collins D. The fourth dimension: A motoric perspective on the anxiety-performance relationship. Int Rev Sport Exerc Psychol. 2016;9(1):1-21.

30. Boo SH, Noh SE, Lee ES et al. Cardiopulmonary response during mechanical horseback riding exercise in healthy adult. J of RWEAT. 2016; 10(4):243-9.

31. Kim MJ, Kim KS, Kim TY et al. The effect of robot based horse riding simulator in perceived psychological factors and energy expenditure for the elderly. Asian J Kinesiol. 2015;17(4):21-9.

32. Sim YS, Lee JH, Lee WY et al. Spirometry and bronchodilator test. Tuberc Respir Dis. 2017;80(2):105-12.

33. Monterroso C, Cruz LS et al. Forced vital capacity and gas exchange parameters during exercise testing in obese patients with dyspnea. Am J Respir Crit Care Med. 2017:A2553.

34. Aruin AS, Kanekar N, Lee YJ et al. Enhancement of anticipatory postural adjustments in older adults as a result of a single session of ball throwing exercise. Exp Brain Res. 2015;233(2):649-55. 